\title{
Nonlinear Optical Responses Of
}

\section{Self-Assembled Monolayers Functionalized With Indolino-Oxazolidine Photoswitches}

Claire Tonnelé,,$+\dagger$ Kornelia Pielak,,,+$\rfloor$ Jean Deviers, ${ }^{\dagger}$ Luca Muccioli, ${ }^{\llbracket}$ Benoit Champagne, ${ }^{*, \neq}$ and Frédéric Castet ${ }^{*, \dagger}$

$\dagger$ †nstitut des Sciences Moléculaires (ISM, UMR CNRS 5255), University of Bordeaux, 351

Cours de la Libération, 33405 Talence, France

$\ddagger$ Unité de Chimie Physique Théorique et Structurale, Chemistry Department, Namur

Institute of Structured Matter, University of Namur, Belgium

ФDepartment of Industrial Chemistry "Toso Montanari", University of Bologna, Viale Risorgimento 4, 40136 Bologna, Italy

E-mail: claire.tonnele@u-bordeaux.fr; benoit.champagne@unamur.be;

frederic.castet@u-bordeaux.fr 


\begin{abstract}
A computational approach combining in sequence molecular dynamic simulations and density functional theory (DFT) calculations is implemented to evaluate the secondorder nonlinear optical (NLO) responses of photoresponsive self-assembled monolayers (SAMs) based on indolino-oxazolidine molecular switches. These numerical simulations provide a complete atomistic picture of the morphology of the SAMs, revealing a high degree of positional disorder and an almost isotropic orientation of the chromophores. Subsequent DFT calculations, carried out to evaluate the average first hyperpolarizability of indolino-oxazolidine switches within the SAM, predict that the structural disorder does not significantly reduce the NLO contrast compared to that of the isolated molecules. Chromophores in the SAM can assume a limited number of specific conformers, due to the high rotational barrier that characterize the conjugated bonds along the indolino/oxazolidine-dyene-thiophene sequence. A notable exception is the rotation about the thiophene-thioalkyl bond, which is not only almost free, but also strongly correlated with the magnitude of the first hyperpolarizability. Controlling this rotation by chemical design could thus be a viable strategy to optimize the SAMs NLO response and the performance of photoresponsive devices based on indolino/oxazolidine switches.
\end{abstract}

\title{
Introduction
}

Organic photochromic compounds have been extensively studied in the last decades, owing to their possible integration into a large variety of photonic and optoelectronic applications. ${ }^{1-3}$ Surface functionalization with molecular photoswitches yields $2 \mathrm{D}$ materials responding to light in a controlled way. ${ }^{4}$ One of the most versatile approaches for surface engineering and interface design is to use self-assembled monolayers (SAMs), ${ }^{5}$ in which highly efficient and selective "click" reactions can be exploited to introduce a wide range of organic functionalities on well-defined base monolayers, typically consisting in azide-terminated alkylsilanes 
grafted on commercially available silica substrates. ${ }^{6-8}$ Thus, the design of photoresponsive SAMs opens the way for virtually endless materials for applications in optical data storage and signal processing.

While effective in writing the information by switching the chromophores between one (meta)stable state to another, the use of linear absorption spectroscopy to probe the system often results in a destructive readout process, since the state of the photochromic molecules can be altered upon irradiation. Exploiting second-order nonlinear optical (NLO) properties instead provides a strategy for non-destructive readout ability to the system, since NLO responses are revealed using near-infrared wavelengths not energetic enough to trigger uncontrolled photoconversions. Molecules exhibiting large and photoswitchable first hyperpolarizability are thus ideal candidates for photonic technologies with multiple storage and non-destructive readout capacity. ${ }^{9-11}$

Filling the gap between isolated molecular switches and photoresponsive materials nevertheless remains a quite challenging task, which requires anchoring the molecular photochromes on a $2 \mathrm{D}$ substrate while preserving i) the reversibility of the switching process, and ii) the NLO responses of the switching states, as well as their contrast. Indeed experiments showing effective photoswitchable NLO SAMs based on fulgimide or azobenzene derivatives have been recently reported, ${ }^{12,13}$ but addressing the aforementioned challenges from a computational point of view has never been tackled. Yet, by offering a microscopic picture of the relationships between the supramolecular structure and NLO properties of the system, numerical simulations have the potential of providing highly useful guidelines to rationally control the NLO responses of the SAMs.

The present work constitutes a first attempt towards the theoretical design of such complex architectures, focusing on indolino-oxazolidine (IND) compounds anchored onto an amorphous $\mathrm{SiO}_{2}$ surface. IND derivatives (Scheme 1) exhibit reversible photo-conversion between a colorless closed form $(\mathrm{CF})$ and a colored open form (OF) associated with large changes in the first hyperpolarizability. ${ }^{14-16}$ To mimic the experimental preparation of indolino- 
oxazolidine SAMs by click chemistry, ${ }^{6}$ IND units were anchored to the silica surface using a two-step process, in which the $\mathrm{SiO}_{2}$ surface is first grafted with azidoalkyl linkers, and subsequently some of the linkers are functionalized with photoswitchable IND units (Scheme 1). Then, extending the framework used for describing the NLO properties of indolinooxazolidines in chloroform solutions, ${ }^{17}$ a sequential approach combining classical molecular dynamics (MD) and quantum mechanics (QM) was employed to evaluate the dynamical behavior and second harmonic generation (SHG) responses of the photoresponsive SAMs. MD simulations gave access to the morphology of the NLO-active layer at the atomistic level, and allowed the exhaustive statistical sampling of the multiple geometrical conformations spanned by the chromophores. Finally, DFT calculations performed on individual molecular fragments extracted from the MD trajectories provided a molecular interpretation on how dynamical geometry distortions, induced by thermal and steric effects, impact the SHG responses of both states of the SAM.

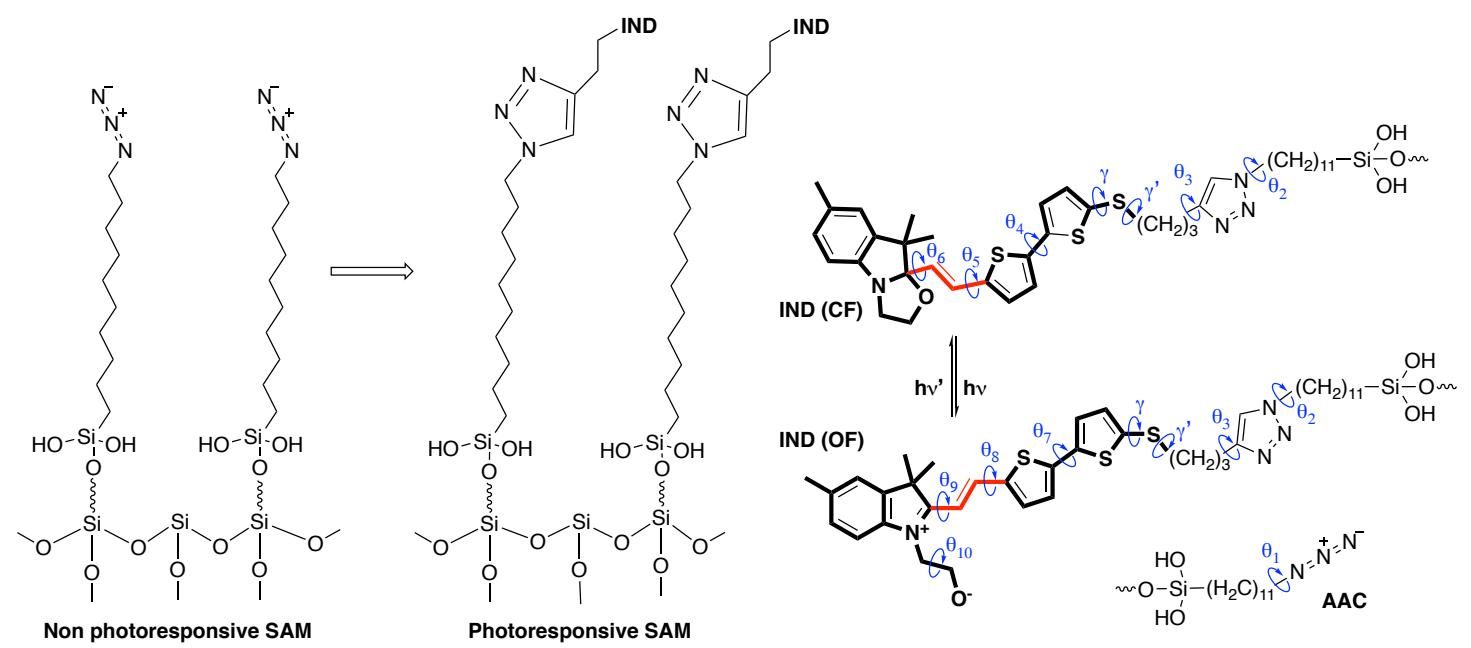

Scheme 1: $\mathrm{SiO}_{2}$ surface functionalized with azidoalkyl chains (left) and with the photoresponsive molecules (middle). A similar scheme was adopted for constructing the SAM geometries in the MD simulations. Right: photochromic reaction between the closed form $(\mathrm{CF})$ and open form (OF) of the indolino-oxazolidine derivative (IND, in bold), and azidoalkyl chain (AAC). FF parameters have been derived for torsions $\theta_{1}$ to $\theta_{10}$ (see ESI for the definition of the dihedral angles). 


\section{Theoretical methodology}

\section{Molecular dynamics simulations}

As detailed in the electronic supporting information (ESI), modified versions of the General AMBER Force Field ${ }^{18}$ (FF) were used to specifically reproduce the equilibrium geometries of the azidoalkyl linkers and of the complete NLO molecule (Scheme 1). Distinct sets of parameters were derived for describing the open and closed forms of IND. Electrostatic potential-fitted (ESP) atomic charges and torsional potentials around the $\theta_{1}-\theta_{10}$ dihedrals were calculated at the M06/6-311G(d) level, and inserted in the force field with the methodology described in reference ${ }^{19}$. In addition, since reliable simulations of NLO responses of push-pull systems require an accurate description of the $\pi$-electron conjugation, ${ }^{16,17}$ AMBER FF bond lengths were adjusted for both states of the switch so as to closely reproduce DFT bond lengths at the corresponding equilibrium geometries (within a maximum absolute error of $0.006 \AA$ ), as well as the bond length alternation (BLA) along the central vinyl bridge (red segments in Scheme 1). The amorphous $\mathrm{SiO}_{2}$ surface of dimensions $52.044 \times$ $51.119 \AA^{2}$ and thickness of about $30 \AA$ was constructed following the scheme described in SI. IND-functionalized SAMs were subsequently prepared using a strategy mimicking the experimental sample preparation. In a first step, a densely packed SAM of 120 azido-undecylsilane chains, corresponding to a coverage of 4.5 molecules $/ \mathrm{nm}^{2}$, was generated to serve as a basis for grafting the NLO molecules. These long non conjugated linkers are used experimentally to electronically decouple the photochromic units from the substrate. This first sample, referred hereafter to as the "non photoresponsive SAM" (see Scheme 1), was equilibrated for 80 ns keeping fixed the position of the grafting oxygen of each azidoalkyl chain. In the second step, imitating the click reaction used in the experimental process, azide terminal groups were replaced by the photochromic compound in its closed form. Since the IND unit is bulkier than the azide unit of the non photoresponsive SAM, only a fraction of azides was replaced, yielding to three samples with different relative IND to total SAM molecule 
ratios, namely $1 / 4,1 / 2$ and $3 / 4$, corresponding to IND coverages of $1.13,2.26$ and 3.38 molecules $/ \mathrm{nm}^{2}$, respectively. The samples were first equilibrated for $30 \mathrm{~ns}$, before we focused on the most dense SAM for which simulation was extended up to 150 ns. Finally, to obtain the open form layer for the highest coverage sample, the force field parameters were switched from the ones optimized for the $\mathrm{CF}$ to the ones optimized for the OF for all chromophores simultaneously, and the resulting OF system was equilibrated for further 85 ns.

\section{Quantum chemical calculations}

Individual molecular structures were extracted from the SAM with the highest relative coverage $(3 / 4)$, at regular intervals along the MD trajectories, and their NLO responses subsequently calculated. Preliminary investigations demonstrated that, in addition to the photoresponsive IND unit, the triazole moiety, and to a lesser extent the alkyl chain, should be explicitly included in the calculations for an accurate evaluation of the hyperpolarizabilities, while the $\mathrm{Si}(\mathrm{OH})_{2} \mathrm{O}$ anchoring unit can be omitted (see SI for details). A total of 900 structures were then used to sample the multiple geometrical conformations adopted by the NLO switches, in both their closed and open forms.

The static and dynamic components of the molecular first hyperpolarizability tensor $(\beta)$ were calculated using time-dependent (TD) DFT with the M06-2X ${ }^{20}$ exchange-correlation functional $(\mathrm{XCF})$ and the $6-311 \mathrm{G}(\mathrm{d})$ basis set. As demonstrated in previous theoretical reports, M06-2X is reliable to calculate hyperpolarizabilities of conjugated organic dyes, ${ }^{21-23}$ including indolino-oxazolidine derivatives, ${ }^{16}$ owing to its substantial amount (54\%) of longrange Hartree-Fock exchange. An incident wavelength of 1064 nm, typical of Nd:YAG laser sources, was used in frequency-dependent SHG calculations. The interfacial NLO responses were analyzed by considering the norm of the first hyperpolarizability vector:

$$
\|\vec{\beta}\|=\beta=\sqrt{\beta_{x}^{2}+\beta_{y}^{2}+\beta_{z}^{2}}
$$


where the $i^{\text {th }}$ component of the $\beta$ vector is defined from the $\beta$ tensor elements:

$$
\beta_{i}=\beta_{i i i}+\frac{1}{3} \sum_{j \neq i}\left(\beta_{i j j}+\beta_{j i j}+\beta_{j j i}\right)
$$

As in the previous work of Tegeder and co., ${ }^{12}$ the anisotropy of the NLO response was also characterized using the component of the $\beta$ vector normal to the surface plane $\left(\beta_{z}\right)$, and the corresponding in-plane ones:

$$
a=\frac{\left|\beta_{z}\right|}{\sqrt{\beta_{x}^{2}+\beta_{y}^{2}}}
$$

All calculations were performed using the Gaussian09 package. ${ }^{24}$ All $\beta$ values are reported assuming a Taylor series expansion of the induced dipole with respect to the applied electric field (T convention), ${ }^{25,26}$ and are given in atomic units $\left(1\right.$ au of $\beta=3.6310^{-42} \mathrm{~m}^{4} \mathrm{~V}^{-1}=$ $\left.3.2063 \times 10^{-53} \mathrm{C}^{3} \mathrm{~m}^{3} \mathrm{~J}^{-2}=8.641 \times 10^{-33} \mathrm{esu}\right)$.

\section{Results and discussion}

\section{Morphology of the self-assembled monolayers}

The equilibrated morphology of the non photoresponsive SAM (i.e. the surface functionalized with azidoalkyl chains only, see Scheme 1), as well as the morphology of the SAM functionalized with the photochromic units in their closed and open forms (for a $3 / 4$ coverage), are represented by the snapshots in Figure 1. The supramolecular organization of the non photoresponsive SAM is characterized by a thickness of $15 \pm 2 \AA$, an average tilt angle of $26 \pm 7^{\circ}$, a nematic order parameter $\left\langle\mathrm{P}_{2}\right\rangle$ of 0.901 and a roughness of $0.86 \AA$. To verify the extent of positional order in all SAMs, we used the 2D radial distribution function $(\mathrm{RDF})$ in the $(x, y)$ plane, which gives a measure of the probability of finding a molecule at a given position with respect to a reference central molecule (Figure 2). The 2D RDF of the non photoresponsive SAM clearly evidences a hexagonal positional order in the sample (with a lattice parameter $d=4.96 \AA$ ), as already observed for H-terminated alkylsiloxane 
SAMs. ${ }^{27}$ Comparing the top panels of Figure 2 reveals that the hexagonal organization
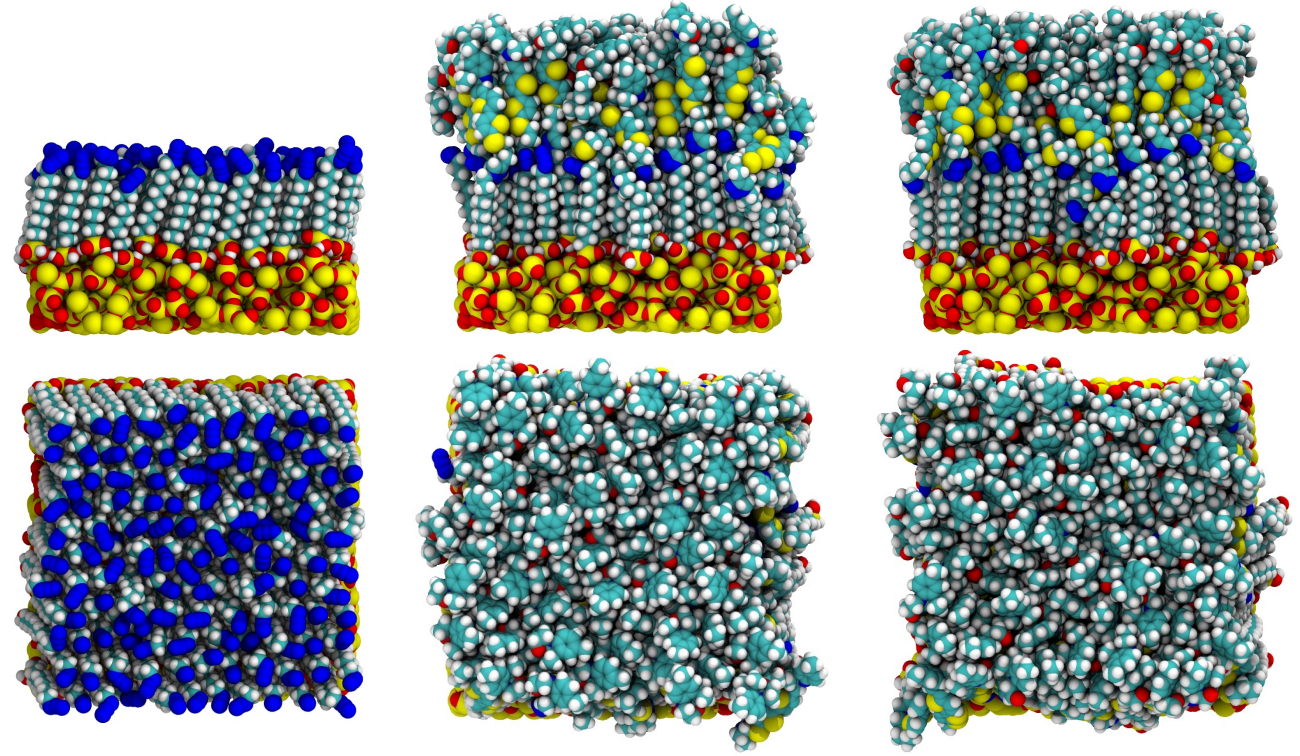

Figure 1: Left: side and top views of the non photoresponsive SAM. Middle: side and top views of the SAM functionalized with the photochromic units in their closed form. Right: in their open form.

of the azidoalkyl layer is globally maintained after functionalization with the photochromic dyes, although it induces a non negligible broadening of the RDF peaks. On the contrary, a visual inspection of the morphology of the photoresponsive SAM (Figure 1) does not evidence any positional order in the top-half sublayer, as confirmed by the 2D RDFs reported in the bottom panels of Figure 2 for the closed and open layers. The distribution of the tilt angle of the dyes with respect to the surface normal (bottom left of Figure 2) reveals that even if a few units are vertically oriented, the distribution is overall almost isotropic. Conversely, the azido-alkyl chains have a uniform, nearly vertical orientation, which is not tilted, unlike that found in the non photoresponsive SAM (cf. top panels of Figure 1).

Decreasing the relative coverage induces a decrease of the SAM thickness, from $40.4 \AA(3 / 4$ coverage) to $34.0 \AA(1 / 2$ coverage) and $26.7 \AA$ (1/4 coverage). Substantiated by a corresponding increase of the tilt (Table SI-1), this demonstrates how NLO molecules bend at low coverage ratio, while they adopt more elongated conformations in the denser layer. On the other hand, the CF to OF photoconversion does not significantly change the thickness 

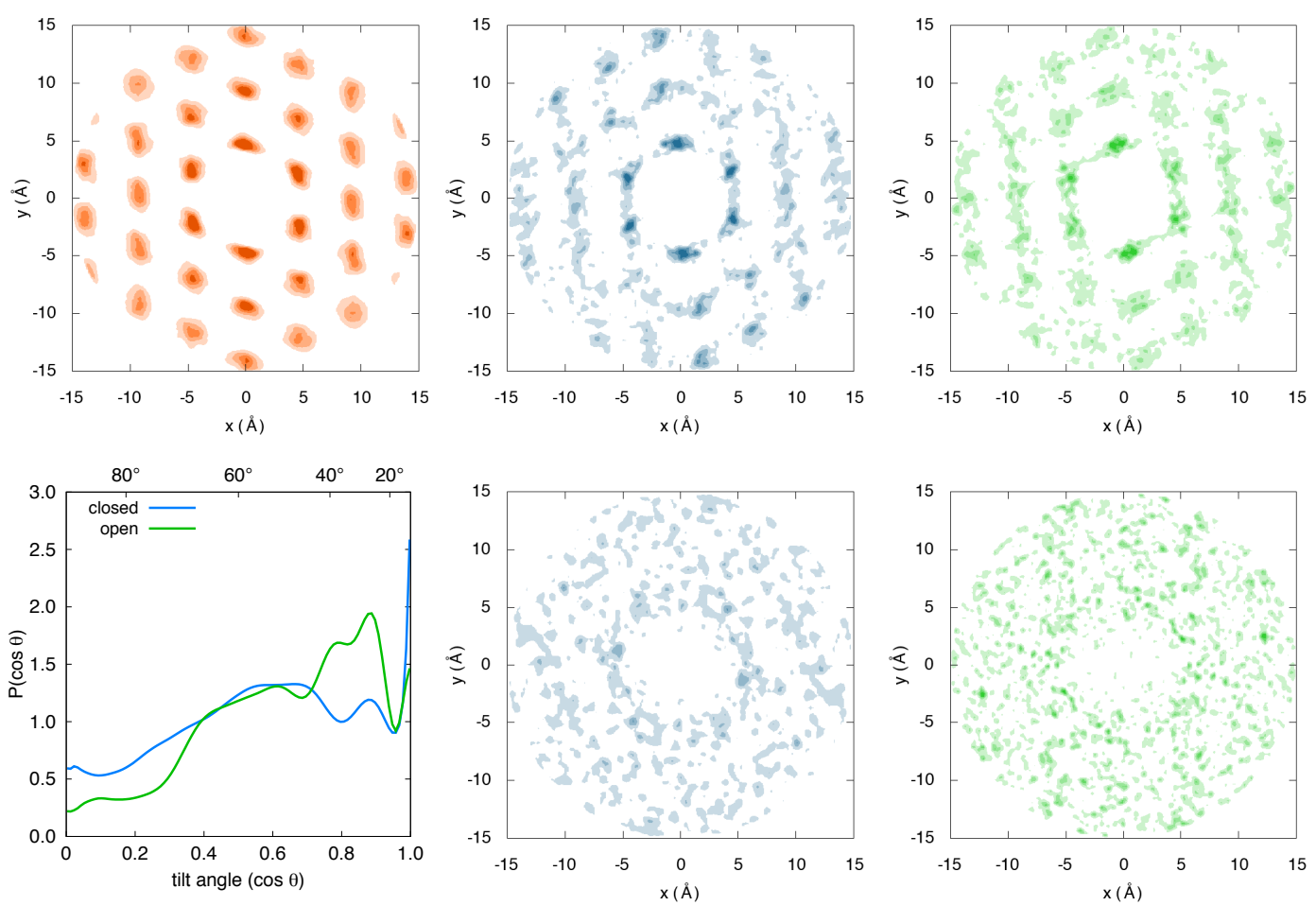

Figure 2: Top: 2D RDF of the centers of mass of the SAM alkyl chains in the non photoresponsive SAM (left), in the photoresponsive SAM in closed form (middle) and in open form (right). Bottom: distribution of the cosine of the tilt angle $\theta$ between the double $\mathrm{C}=\mathrm{C}$ bond of the vinyl bridge and the axis normal to the surface plane (left), and 2D RDF of the centers of mass of the triazole-indolino-oxazolidine units in the closed and open form SAMs (middle and right panels).

of the SAM (40.4 vs. $40.6 \AA$ for CF and OF, respectively). Reversely, the roughness slightly increases when decreasing the coverage, from 1.32 to $3.15 \AA$.

Focusing on the SAM with higher IND density (3/4 coverage), we investigated more closely the geometrical conformations spanned by the photochromic molecules onto the surface. Consistently with the DFT geometries used for the force field parameterization, the average BLA of the open forms is three times smaller $(0.089 \pm 0.061 \AA)$ than that of closed forms $(0.288 \pm 0.061 \AA)$. The distributions of the torsional angles adopted by the molecules within the SAM, plotted with respect to the corresponding rotational potentials of isolated molecules (Figures SI-8 and SI-9), are also informative. Due to high rotation barriers (vary- 
ing from 3.2 to $13.0 \mathrm{kcal} / \mathrm{mol}$ ), the population of the $\theta_{4}-\theta_{10}$ and $\gamma^{\prime}$ dihedrals (Scheme 1) concentrate around the minima of the potential energy curves of the isolated molecules. Reversely, the $\theta_{1}-\theta_{3}$ and $\gamma$ dihedrals exhibit very broad distributions due to low energy barriers ( $\sim 1 \mathrm{kcal} / \mathrm{mol}$ or smaller). Interestingly, the maxima of the distributions of these torsional angles do not always correspond exactly to minima of the potential energy curve, suggesting that the most probable values are dictated by intermolecular interactions.

\section{Commutation of the nonlinear optical responses}

The statistical distributions of the static and dynamic first hyperpolarizabilities ( $\beta$, eq. 1), of their $z$-component $\left(\beta_{z}\right)$, and of the anisotropy factors (a, eq. 3) are illustrated in Figure 3, while average values and standard deviations $(\sigma)$ are collected in Table 1 . The interquartile ranges (IQR), which provide a robust measure of the dispersion of statistical distributions, are also reported.

Considering first the static responses, dynamical geometry fluctuations and steric interactions lead to broad $\beta$ and $\beta_{z}$ distributions, characterized by large $\sigma$ and IQR values. It also clearly appears from Figure 3 that the distributions of open forms are much broader than those of closed forms, as confirmed by IQR values about four times larger. Moreover for both forms the component normal to the surface plane, $\beta_{z}$, dominates the static hyperpolarizability, as indicated by average anisotropy ratios $\langle a\rangle$ lying between 3.6 and 4.2. Interestingly, a similar $\langle a\rangle$ value $(5.70 / \sqrt{2}=4.03)$ was deduced from recent experimental measurements and DFT calculations for the trans-configuration of azobenzene-based SAMs. ${ }^{12}$ However, while the trans-cis isomerization in azobenzene derivatives was shown to strongly reduce the anisotropy of the NLO response owing to the realignment of the upper phenyl ring parallel to the surface, the oxazolidine ring opening in IND derivatives hardly changes the anisotropy of the SHG

response, consistently with the invariance of the SAM thickness mentioned above. One also notes that, contrary to the absolute NLO responses, the distribution of the anisotropy ratios is slightly less dispersed for the open form, even if in both cases a considerable fraction of 
molecules has very low anisotropy, consistently with the broad distributions of tilt angles shown in Figure 2.

The commutation between the closed and open forms induces a large enhancement of the static first hyperpolarizability, as shown by the shift of the $\beta$ distributions. The $\mathrm{OF} / \mathrm{CF}$ NLO contrast, evaluated as the ratio between the mean values of the distributions (Table 1 ), is equal to $\sim 7$. Strikingly, this contrast is only slightly smaller than the one calculated for the isolated molecule in its equilibrium geometry $(\beta(\mathrm{POF}) / \beta(\mathrm{CF})=8.93)$. This result indicates that, despite the close molecular packing induces strong geometrical distortions, the high degree of disorder of the SAM is not detrimental to the average NLO contrast, which is of crucial importance for device efficiency. This also confirms and extends to amorphous morphologies the conclusions previously deduced from periodic self-consistent charge density functional tight binding calculations, that single IND units anchored through silanol linkers onto a $\mathrm{SiO}_{2}$ surface maintain their NLO activities and contrasts. ${ }^{28}$

Considering the dynamic NLO responses, the average values of $\beta$ and $\beta_{z}$ computed for the closed forms are enhanced by a factor 2.2 compared to their static analogues. The dynamical distributions are also more dispersed than the static ones, with IQR values about two times larger. In the case of open forms, the dynamic $\beta$ values are spread over a very broad range, the NLO responses of a few set of structures being strongly contaminated by electronic excitation resonances. These resonance phenomena give rise to numerical instabilities, which make the calculated dynamic $\beta$ values of the OF less reliable. To partially circumvent this problem, the averages of the dynamic NLO properties of the OF were estimated by considering only the molecules with $\beta$ values within the interquartile range. For the open form, the enhancement due to frequency dispersion effects, defined as the ratio between the dynamic and static responses, amounts to 7.5 and 3.5 for $\beta$ and $\beta_{z}$, respectively. Interestingly, the anisotropy of the NLO responses is not impacted by frequency resonance, the average anisotropy ratio $\langle a\rangle$ being identical to that calculated considering the static responses. Finally, the dynamic $\beta(\mathrm{OF}) / \beta(\mathrm{CF}) \mathrm{NLO}$ contrast amounts to $\sim 26$, i.e. more than 3 times the static one, while 
it is reduced to $\sim 13$ when considering the $z$-component only.
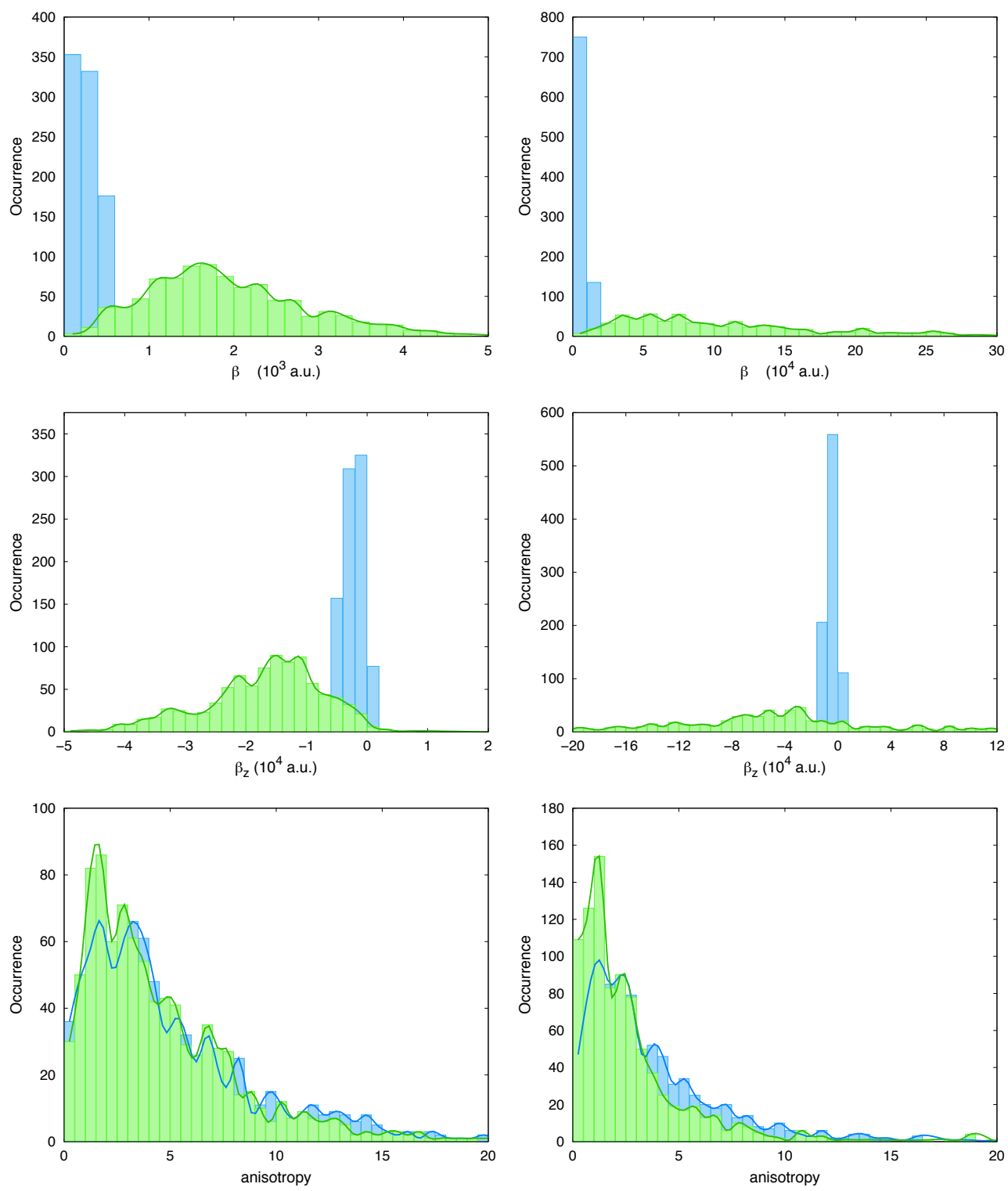

Figure 3: Distributions of the static (left) and dynamic (right) values of $\beta$ (top), $\beta_{z}$ (middle) and $a$ (bottom), for closed (blue) and open (green) forms.

\section{Relationships between geometrical structures and NLO responses}

To gain a deeper understanding on the impact of the geometrical distortions on the intrinsic (static) NLO properties of the photochromes, correlations between the calculated $\beta$ responses and molecular structural parameters were further analyzed. The variations of $\beta$ 
Table 1: Averaged values, standard deviations $(\sigma)$, and interquartile range (IQR) of the average static $(\lambda=\infty)$ and dynamic $(\lambda=1064 \mathbf{n m})$ hyperpolarizabilities $(\beta)$, of their $z$-component $\left(\beta_{z}\right)$, and of the anisotropy factor $(a)$. The two last lines report the $\mathrm{OF} / \mathrm{CF}$ enhancement ratios of the NLO responses.

\begin{tabular}{lcccc}
\hline & \multicolumn{2}{c}{$\lambda=\infty$} & \multicolumn{2}{c}{$\lambda=1064 \mathrm{~nm}$} \\
\hline Property & $\mathrm{CF}$ & $\mathrm{OF}$ & $\mathrm{CF}$ & $\mathrm{OF}$ \\
\hline$\langle\beta\rangle \pm \sigma_{\beta}$ & $2624 \pm 1655$ & $19473 \pm 11410$ & $5699 \pm 3942$ & $(146337 \pm 70035)^{a}$ \\
$\left\langle\beta_{z}\right\rangle \pm \sigma_{\beta_{z}}$ & $-2311 \pm 1765$ & $-16725 \pm 11322$ & $-4960 \pm 4176$ & $(-59100 \pm 123161)^{a}$ \\
$\langle a\rangle \pm \sigma_{a}$ & $4.20 \pm 5.18$ & $3.56 \pm 4.19$ & $4.18 \pm 5.22$ & $(3.54 \pm 11.09)^{a}$ \\
$\mathrm{IQR}(\beta)$ & 2765 & 12036 & 6309 & 275013 \\
$\mathrm{IQR}\left(\beta_{z}\right)$ & 2770 & 11940 & 6359 & 188310 \\
$\mathrm{IQR}(a)$ & 3.62 & 3.25 & 3.66 & 2.38 \\
$\langle\beta(O F)\rangle /\langle\beta(C F)\rangle$ & \multicolumn{2}{c}{$7.42 \pm 9.03$} & \multicolumn{2}{c}{$25.68 \pm 30.05$} \\
$\left\langle\beta_{z}(O F)\right\rangle /\left\langle\beta_{z}(C F)\right\rangle$ & \multicolumn{2}{c}{$7.24 \pm 10.43$} & $11.92 \pm 34.86$ \\
\hline \multicolumn{2}{c}{ Calculated by excluding all the molecules with $\beta$} & values outside the IQR.
\end{tabular}

with torsional angles around single bonds are reported in ESI, while Figure 4 illustrates the variations of $\beta$ with respect to $\gamma$, as well as to the BLA along the vinyl bridge connecting the indolino-oxazolidine and the bithiophene. As already observed for molecules in solution, ${ }^{16,17}$ Figure 4 (top panel) clearly evidences that the difference in the magnitude of the $\beta$ response between open and closed forms is mainly driven by the BLA along the central part of the photochromes, whose average value decreases from 0.288 to $0.089 \AA$. Considering $\mathrm{CF}$ and OF separately, it turned out quite unexpectedly that the amplitude of $\beta$ in both forms is strongly correlated with the value of the torsion angle $\gamma$ (see Scheme 1). As illustrated in Figure 4 (middle and bottom panels), large first hyperpolarizabilities are obtained for planar conformations with respect to $\gamma\left(\right.$ i.e. when $\gamma=0^{\circ}$ or $180^{\circ}$ ), while $\beta$ is much smaller when the S-C bond of the thiomethylene group is perpendicular to the thiophene ring. Further analyses detailed in the ESI revealed that upon photoexcitation, planar conformations allow the conjugation of the lone pair of the sulfur atom of the thiopropylene, giving rise to an important charge transfer between the bonded sulfur and the dithiophene. This effect is at the origin of the enhancement of the first hyperpolarizability compared to perpendicular conformations. On the contrary, the distributions of $\beta$ with respect to $\theta_{2}$ and $\theta_{3}$ (see ESI) revealed that the amplitude of the NLO responses is not correlated to rotations around these 

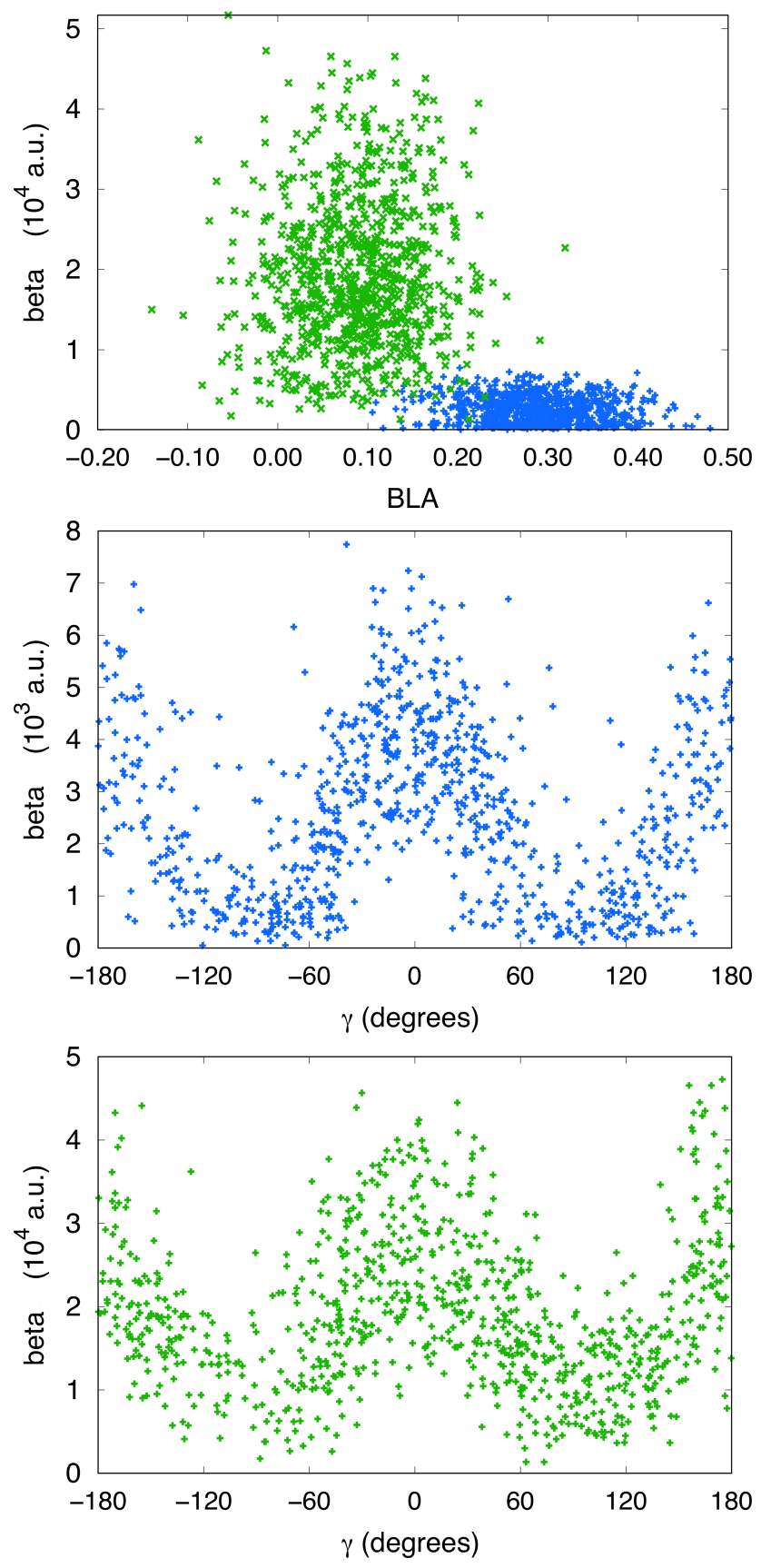

Figure 4: $\beta$ values plotted versus the BLA and $\gamma$ torsion angle in closed (blue) and open (green) forms.

two dihedrals, which also assume all possible values due to low rotational energy barriers. The other dihedrals $\left(\theta_{4}-\theta_{10}\right)$ adopt instead specific values due to conjugation effects and steric constraints.

The striking correlation between the NLO response and the $\gamma$ torsional angle reveals a 
crucial effect for the chemical engineering of 2D NLO materials. Owing to the low rotational energy barrier for the rotation about $\gamma(\sim 0.8 \mathrm{kcal} / \mathrm{mol}$, see ESI $)$, the conformations adopted are mostly driven by intermolecular contacts. Since these packing effects are very difficult to control, it emerges that the best strategy to maximize the NLO responses of dye-functionalized SAMs consists in designing rigid molecules with geometry constrained to their highest hyperpolarizability conformation.

\section{Conclusions}

In this work, we have used a sequential multiscale approach combining atomistic MD simulations and DFT calculations to evaluate the second harmonic generation responses of photoresponsive SAMs based on indolino-oxazolidine derivatives. MD simulations revealed that photoresponsive regions of the SAMs exhibit a high degree of orientational and positional disorder due to the flexibility of the photoresponsive units (in particular that of the thioalkyl group), which on the one hand is detrimental for crystalline packing, and on the other hand makes the chromophore conformation highly sensitive to intermolecular interactions. Quite interestingly, DFT calculations carried out on a large selection of simulated molecular structures evidence that, despite large geometrical distortions of the photochromic molecules and the lack of orientational order, IND-based SAMs are expected to display a good contrast in first hyperpolarizability upon the photochromic conversion, of the same order of magnitude as that observed in the isolated molecule.

As already known from previous studies, ${ }^{16,17}$ the large NLO enhancement from the closed to the open configuration can be attributed to the increase of the $\pi$-electron conjugation between the indolino-oxazolidine and bithiophene moieties, as described by the strong decrease of the bond length alternation along the vinyl linker connecting these two units. More surprising was the correlation found between the magnitude of the first hyperpolarizability in both forms and the $\gamma$ torsional angle around the single bond between bithiophene and 
thioalkyl groups. Since this rotation is quasi barrierless in the isolated molecule, the statistical distribution of this dihedral angle is very broad for both forms of the SAMs studied here. In such a case, only controlling by chemical design the shape of this distribution in newly synthesized indolino-oxazolidine derivatives would allow maximizing their NLO responses. More generally, these results tell us that calculations of the NLO properties of isolated molecules allows a reliable screening of the most effective compounds for subsequent integration in $2 \mathrm{D}$ materials, but also highlight that optimizing the NLO responses of devices requires a fine control of the intermolecular interactions, which induce significant distortions in the molecular structure. Reducing the flexibility of the photoresponsive molecular units, together with variations of the coverage rate or the use of bulky separators, appear as promising strategies.

Mutual polarization effects, not considered in this first study, might also significantly impact the NLO responses of the SAMs. Future investigations, carried out on a representative set of molecular aggregates, are expected to provide design rules to effectively exploit the collective effects for optimizing the amplitude of the NLO responses of the system, as well as their contrast upon commutation. The sequential MD-QM approach implemented here promises to be a highly useful computational tool to explore these different alternatives.

\section{Acknowledgement}

K. P. thanks the Région Aquitaine (INMERON Project, convention 2014-1R10102-00002867) and the University of Namur for co-financing her PhD grant. This work was carried out in the frame of the Centre of Excellence LAPHIA (Investments for the future: Programme IdEx Bordeaux - LAPHIA (ANR-10-IDEX-03-02). It was also supported by funds from the M-ERA.NET project MODIGLIANI (ANR-15-MERA-0002-01) and from the Francqui Foundation. The calculations were performed on the computing facilities of the Consortium des Equipements de Calcul Intensif (CECI, http://www.ceci-hpc.be), and particularly those of the Technological Platform on High-Performance Computing, for which we gratefully 
acknowledge the financial support of the FNRS-FRFC (Conventions No. 2.4.617.07.F and 2.5020.11) and of the University of Namur, on Zenobe, the Tier-1 facility of the Walloon Region (Convention 1117545), as well as on the Mésocentre de Calcul Intensif Aquitain (MCIA) of the University of Bordeaux, financed by the Conseil Régional d'Aquitaine and the French Ministry of Research and Technology. L.M, B.C. and F.C thank Prof. Vincent Rodriguez for helpful discussions.

\section{Supporting Information Available}

Force field parameterization; Preparation of the $\mathrm{SiO}_{2}$ surface; Functionalized surfaces with various coverage rates; Choice of the relevant molecular fragment for NLO calculations; Probability distributions of dihedrals; Relationships between geometrical structures and NLO responses. This material is available free of charge via the Internet at http://pubs acs . org/.

\section{References}

(1) Feringa, W. R., B. L.; Browne, Ed. Molecular Switches, Second Edition; Wiley-VCH, Weinheim, 2011.

(2) Orgiu, E.; Samorì, P. 25th Anniversary Article: Organic Electronics Marries Photochromism: Generation of Multifunctional Interfaces, Materials, and Devices. Adv. Mater. 2014, 26, 1827-1845.

(3) Andréasson, J.; Pischel, U. Molecules with a Sense of Logic: a Progress Report. Chem. Soc. Rev. 2015, 44, 1053-1069.

(4) Browne, W. R.; Feringa, B. L. Light Switching of Molecules on Surfaces. Annu. Rev. Phys. Chem. 2009, 60, 407-428. 
(5) Casalini, S.; Bortolotti, C. A.; Leonardi, F.; Biscarini, F. Self-assembled monolayers in organic electronics. Chem. Soc. Rev. 2017, 46, 40-71.

(6) Lummerstorfer, T.; Hoffmann, H. Click Chemistry on Surfaces: 1,3-Dipolar Cycloaddition Reactions of Azide-Terminated Monolayers on Silica. J. Phys. Chem. B 2004, $108,3963-3966$.

(7) Haensch, C.; Hoeppener, S.; Schubert, U. S. Chemical modification of self-assembled silane based monolayers by surface reactions. Chem. Soc. Rev. 2010, 39, 2323-2334.

(8) Nicosia, C.; Huskens, J. Reactive self-assembled monolayers: from surface functionalization to gradient formation. Mater. Horiz. 2014, 1, 32-45.

(9) Coe, B. J. Molecular Materials Possessing Switchable Quadratic Nonlinear Optical Properties. Chem. Eur. J. 1999, 5, 2464-2471.

(10) Asselberghs, I.; Clays, K.; Persoons, A.; Ward, M. D.; McCleverty, J. Switching of Molecular Second-Order Polarizability in Solution. J. Mater. Chem. 2004, 14, 28312839.

(11) Castet, F.; Rodriguez, V.; Pozzo, J.-L.; Ducasse, L.; Plaquet, A.; Champagne, B. Design and Characterization of Molecular Nonlinear Optical Switches. Acc. Chem. Res. 2013, $46,2656-2665$.

(12) Schulze, M.; Utecht, M.; Moldt, T.; Przyrembel, D.; Gahl, C.; Weinelt, M.; Saalfrank, P.; Tegeder, P. Nonlinear optical response of photochromic azobenzenefunctionalized self-assembled monolayers. Phys. Chem. Chem. Phys. 2015, 17, 1807918086.

(13) Schulze, M.; Utecht, M.; Hebert, A.; Rück-Braun, K.; Saalfrank, P.; Tegeder, P. Reversible Photoswitching of the Interfacial Nonlinear Optical Response. J. Phys. Chem. Lett. 2015, 6, 505-509. 
(14) Sanguinet, L.; Pozzo, J. L.; Rodriguez, V.; Adamietz, F.; Castet, F.; Ducasse, L.; Champagne, B. Acido- and Photo-triggered NLO Properties Enhancement. J. Phys. Chem. B 2005, 109, 11139-11150.

(15) Mançois, F.; Pozzo, J.-L.; Pan, J.; Adamietz, F.; Rodriguez, V.; Ducasse, L.; Castet, F.; Plaquet, A.; Champagne, B. Two-Way Molecular Switches with Large Nonlinear Optical Contrast. Chem.-Eur. J. 2009, 15, 2560-2571.

(16) Pielak, K.; Bondu, F.; Sanguinet, L.; Rodriguez, V.; Champagne, B.; Castet, F. SecondOrder Nonlinear Optical Properties of Multiaddressable Indolinooxazolidine Derivatives: Joint Computational and Hyper-Rayleigh Scattering Investigations. J. Phys. Chem. C 2017, 121, 1851-1860.

(17) Pielak, K.; Tonnelé, C.; Sanguinet, L.; Cariati, E.; Righetto, S.; Muccioli, L.; Castet, F.; Champagne, B. Dynamical behavior and second harmonic generation responses in acido-triggered molecular switches. Submitted 2018,

(18) Wang, J.; Wolf, R. M.; Caldwell, J. W.; Kollman, P. A.; Case, D. A. Development and testing of a general amber force field. J. Comput. Chem. 2004, 25, 1157-1174.

(19) Pizzirusso, A.; Pietro, M. E. D.; Luca, G. D.; Celebre, G.; Longeri, M.; Muccioli, L.; Zannoni, C. Order and Conformation of Biphenyl in Cyanobiphenyl Liquid Crystals: A Combined Atomistic Molecular Dynamics and 1H NMR Study. ChemPhysChem 2014, $15,1356-1367$.

(20) Zhao, Y.; Truhlar, D. G. The M06 Suite of Density Functionals for Main Group Thermochemistry, Thermochemical Kinetics, Noncovalent Interactions, Excited States, and Transition Elements: two New Functionals and Systematic Testing of Four M06-Class Functionals and 12 Other Functionals. Theor. Chem. Acc. 2008, 120, 215-241.

(21) de Wergifosse, M.; Champagne, B. Electron correlation effects on the first hyperpolarizability of push-pull $\pi$-conjugated systems. J. Chem. Phys. 2011, 134, 074113. 
(22) Johnson, L. E.; Dalton, L. R.; Robinson, B. H. Optimizing Calculations of Electronic Excitations and Relative Hyperpolarizabilities of Electrooptic Chromophores. Acc. Chem. Res. 2014, 47, 3258-3265.

(23) Garrett, K.; Sosa Vazquez, X.; Egri, S. B.; Wilmer, J.; Johnson, L. E.; Robinson, B. H.; Isborn, C. M. Optimum Exchange for Calculation of Excitation Energies and Hyperpolarizabilities of Organic Electro-optic Chromophores. J. Chem. Theory Comput. 2014, 10, 3821-3831.

(24) Frisch, M. J.; Trucks, G. W.; Schlegel, H. B.; Scuseria, G. E.; Robb, M. A.; Cheeseman, J. R.; Scalmani, G.; Barone, V.; Mennucci, B.; Petersson, G. A. et al. Gaussian 09 revision D01. Gaussian Inc. Wallingford CT 2009.

(25) Willetts, A.; Rice, J. E.; Burland, D. M.; Shelton, D. P. Problems in the comparison of theoretical and experimental hyperpolarizabilities. J. Chem. Phys. 1992, 97, 75907599 .

(26) Reis, H. Problems in the comparison of theoretical and experimental hyperpolarizabilities revisited. J. Chem. Phys. 2006, 125, 014506.

(27) Roscioni, O. M.; Muccioli, L.; Mityashin, A.; Cornil, J.; Zannoni, C. Structural Characterization of Alkylsilane and Fluoroalkylsilane Self-Assembled Monolayers on $\mathrm{SiO}_{2}$ by Molecular Dynamics Simulations. J. Phys. Chem. C 2016, 120, 14652-14662.

(28) Nénon, S.; Champagne, B. SCC-DFTB calculation of the static first hyperpolarizability: From gas phase molecules to functionalized surfaces. J. Chem. Phys. 2013, 138, 204107. 
Graphical TOC Entry 